\title{
Prognostic Implications of Arginase and Cytokeratin 19 Expression in Hepatocellular Carcinoma After Curative Hepatectomy: Correlation With Recurrence-Free Survival
}

\author{
Ifeyinwa Emmanuela Obioraha, b, Joeffrey Chahine ${ }^{\mathrm{a}}$, Kyungmin Ko ${ }^{\mathrm{a}}$, \\ Byoung Uk Park ${ }^{\mathrm{a}}$, Jose deGuzman ${ }^{\mathrm{a}}$, Bhaskar Kallakury ${ }^{\mathrm{a}}$
}

\begin{abstract}
Background: The prognostic value of arginase expression in hepatocellular carcinoma (HCC) has been evaluated previously. However, no clear distinction exists yet on the role of arginase-1 as a predictor of recurrence in HCC. Cytokeratin 19 (CK19), a cholangiocytic marker, is occasionally expressed in $\mathrm{HCC}$, but the combination of arginase-1 and CK19 expression has never been evaluated. The aim of the study was to investigate the usefulness of arginase- 1 and CK19 expression alone and in combination for prognosticating $\mathrm{HCC}$ tumor recurrence after surgical resection.
\end{abstract}

Methods: Tissue sections from 112 HCCs were immunostained using an automated method and the mouse monoclonal arginase- 1 and mouse monoclonal CK19 antibodies. The clinicopathologic variables, including alpha-fetoprotein levels, viral hepatitis, cirrhosis, tumor size, grade and number, vascular invasion, tumor-node-metastasis (TNM) stage, and tumor recurrence and survival, were obtained from each patient's medical records. The variables were assessed for correlation with the immunochemical results. Comparisons of recurrence-free and overall survival were performed using univariate and multivariate regression analyses. A P-value of $\leq 0.05$ was considered statistically significant.

Results: High arginase-1 expression was detected in the HCCs of 93 patients (83\%), whereas CK19 was positive in the HCCs of only 19 patients $(17 \%)$. In the univariate analyses, CK19 positivity in HCC was associated with decreased recurrence-free survival compared with CK19-negative HCC $(\mathrm{P}=0.0002)$. Arginase-1 expression was associated with decreased recurrence-free survival when patients were stratified over advanced TNM stage and presence of vascular invasion. The combination of arginase- 1 and CK19 expression was a

Manuscript submitted February 8, 2019, accepted March 18, 2019

aDepartment of Pathology, Medstar Georgetown University Hospital, 3800 Reservoir Road NW, Washington, DC 20007, USA

${ }^{b}$ Corresponding Author: Ifeyinwa E. Obiorah, Department of Pathology, Medstar Georgetown University Hospital, 3800 Reservoir Road NW, Washington, DC 20007, USA. Email: Ifeyinwa.e.obiorah@gunet.georgetown.edu

doi: https://doi.org/10.14740/gr1156 better predictor of decreased recurrence-free survival $(\mathrm{P}=0.00008)$. Arginase-1/CK19 expressions when combined with multiple tumors, TNM stage and vascular invasion were also associated with decreased recurrence-free survival. In the multivariate analysis, tumor grade, CK19 and arginase-1/CK19 expressions were identified as independent prognostic indicators for decreased recurrence-free survival.

Conclusion: Arginase-1 and CK19 combination immunoreactivity is a potential biomarker of adverse prognosis in HCC, correlating with the presence of multiple tumors, vascular invasion and advanced stage.

Keywords: Hepatocellular carcinoma; Liver; Arginase; Surgery; Differentiation; Cytokeratin 19; Recurrence; Survival

\section{Introduction}

Curative surgery remains the mainstay of treatment for hepatocellular carcinoma (HCC) in patients with non-metastasized tumors. Recurrence of the neoplasm following the curative hepatectomy can greatly impact the long-term prognosis of those HCC patients. The clinicopathologic risk factors of tumor size, tumor number, vascular invasion and tumor-node-metastasis (TNM) staging have been shown to be associated with the poor clinical outcomes [1-3]; however, the validation of such potential biomarkers for prognosis is still needed.

Arginase-1 is an enzyme that is responsible for the conversion of arginine to urea in the urea cycle and it is highly expressed in benign and malignant hepatocytes, especially those located around the periportal regions $[4,5]$. Compared to other hepatocytic markers, arginase-1 is a highly sensitive and specific marker of hepatocellular differentiation $[6,7]$. The prognostic value of arginase- 1 has only been analyzed in one study to date. Mao and colleagues [8] showed that high arginase-1 expression was associated with better recurrence-free and overall survival; however, their stratification of patients into high and low arginase expression was not clearly elucidated, since $98 \%$ of all the HCC tumors examined expressed arginase-1.

In the fetal liver, hepatic stem cells give rise to hepato- 
blasts, which differentiate into the hepatocytic and biliary lineages. Prior to this differentiation, overexpression of cytokeratin 19 (CK19) is observed in the hepatic progenitor cells $[9,10]$. CK19 expression decreases in cells committed to the hepatocytic pathway, whereas the expression is retained in the cholangiocytes $[9,11]$. Although it is well accepted that CK19 is a biomarker of cholangiocarcinoma, its overexpression has been observed in HCC of hepatic stem cell origin and found to be associated with poor prognosis [12-14].

Previous studies have evaluated the prognostic importance of arginase expression or CK19 expression in HCC patients. Combined detection of both markers has never been evaluated. In this study, we first validated arginase- 1 expression in HCC tissues by using a highly specific monoclonal antibody and then we explored the predictive value of arginase- 1 and CK19 combined expression for the prognosis of HCC following curative surgery.

\section{Materials and Methods}

\section{Patients and tissue specimens}

One hundred twelve patients who underwent curative hepatectomy for HCC at our institution between January 2013 and January 2016 were included. The inclusion criteria identified patients: 1) with histologic diagnosis of HCC; 2) who underwent curative surgery with no presurgical treatment resulting in tissue necrosis; 3) who had formalin-fixed paraffin-embedded tissue samples; 4) with no concurrent presence of another liver carcinoma; and 5) with complete clinicopathologic and follow-up information. Two hundred fifteen patients were initially identified, but 103 of these were excluded due to either previous treatment, concurrent carcinoma (i.e. hepatocholangiocarcinoma) or incomplete follow-up data.

Ages of the 112 total included patients ranged from 16 to 83 years (median: 65 years). Eighty-one (72.3\%) males and 31 $(27.7 \%)$ females were included. Hepatitis $\mathrm{C}$ virus was detected in 61 patients $(54.5 \%)$, and hepatitis B infection was found in 20 patients $(17.8 \%)$. Liver cirrhosis was present in 72 patients (64.3\%).

Serum alpha-fetoprotein (AFP) level had been measured prior to surgery. The tumor size, tumor number, tumor grade and presence of vascular invasion were determined by postoperative pathological examination. The TNM staging was carried out according to the seventh edition system [15]. For all patients, the postoperative follow-up schedule consisted of imaging and AFP level screening at every 3 months for the first 6 months, followed by a 12-month interval thereafter. Diagnosis of recurrence was based on typical imaging appearance in computed tomography and/or magnetic resonance imaging scan with/without an elevated serum AFP level. The data for overall survival and recurrence covered the range of time from each patient's hepatic resection to death or the last clinical observation, respectively. The median follow-up period was 33 months (range: 1 - 67 months).

The clinicopathologic features of the patients in our study population are summarized in Table 1. The study was approved by the Georgetown University Institutional Review Board.

\section{Immunohistochemical evaluation}

Sections (4 $\mu \mathrm{m}$ thick) of the formalin-fixed, paraffin-embedded tissue blocks of all the studied cases (a single HCC sample per patient) were investigated by specific antibody reactivity for the expression of arginase-1 (dilution 1:4,000, clone SL6ARG; Invitrogen, San Diego, CA, USA) and CK19 (ready-to-use, clone RCK108; Dako, Glostrup, Denmark) using an automated method (Dako enVision + dual link systemhorseradish peroxidase). Pretreatment of the formalin-fixed, paraffin-embedded tissue sections with heat-induced epitope retrieval was performed using diluted enVision Flex target retrieval solution, low $\mathrm{pH}(\times 50)(\mathrm{K} 8004$; Dako $)$.

Deparaffinization, rehydration and epitope retrieval were performed by the Dako PT link pretreatment system (PT100/ PT101) using the following parameters: preheat temperature: 85 ${ }^{\circ} \mathrm{C}$; epitope retrieval temperature and time: $97^{\circ} \mathrm{C}$ for $20 \mathrm{~min}$ and cool-down to $65^{\circ} \mathrm{C}$. Racks were placed in diluted enVision Flex wash buffer $(\times 20)(\mathrm{K} 8007$; Dako $)$ for $5 \mathrm{~min}$. The slides were then treated with Flex peroxidase blocking solution (SM801; Dako) for $5 \mathrm{~min}$, followed by incubation with the primary antibodies (arginase-1 or CK19) for $20 \mathrm{~min}$. The slides were next treated sequentially with Flex mouse linker (SM804; Dako) for $15 \mathrm{~min}$, Flex horseradish peroxidase (SM802; Dako) for $20 \mathrm{~min}$, and Flex diaminobenzidine with substrate-chromogen (SM803; Dako) for $10 \mathrm{~min}$. Sections were counterstained with hematoxylin for $5 \mathrm{~min}$ before assessment under microscope.

Patient-matched normal (adjacent benign) liver tissues served as the positive control and normal (adjacent benign) bile duct epithelium served as an internal positive control for CK19 (both processed as described above). The negative control was generated by identical treatment of each patient's experimental tissue but omitting the primary antibody. A positive result for arginase-1 expression was indicated by cytoplasmic staining with or without nuclear staining in the tumor cells. The extent of positive tumor cells was classified as 1 (focal; when $\leq 50 \%$ of the tumor cells were positive), 2 (regional; when $50-90 \%$ of the tumor cells were positive), and 3 (diffuse; when $\geq 90 \%$ of the tumor cells were positive). The intensity of immunostaining was scored as 0 (negative/weak staining), 1+ (moderate staining), and $2+$ (intense staining). The extent and intensity tumor scores were multiplied to give a composite score (range: 0 - 6) for each tissue specimen. Composite scores of 0 - 3 were described as having low arginase- 1 expression, and scores of 4 - 6 were defined as high arginase- 1 expression. CK19 expression was considered positive if moderate or intense staining was present in $\geq 5 \%$ of the tumor cells [12]. Immunoreactivity was semi-quantitatively scored by two pathologists.

\section{Statistical analysis}

The relationships between arginase-1 and CK19 expressions and the various clinicopathologic characteristics were determined using Fisher's exact test. Comparisons of recurrencefree and overall survival were performed by the log-rank test. 
Table 1. Associations of Arginase-1/CK19 Expression and Clinicopathologic Parameters in HCC

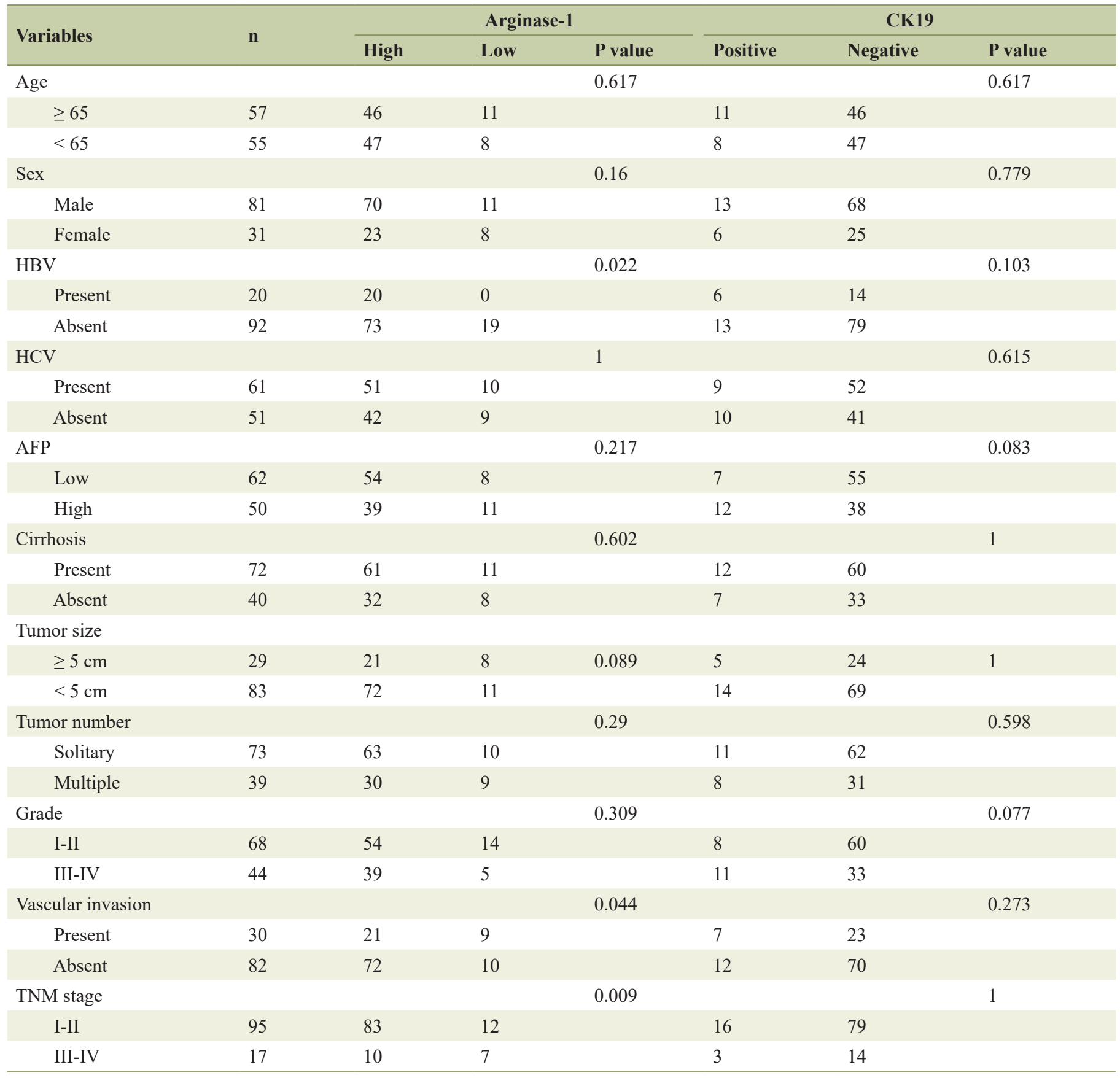

HCC: hepatocellular carcinoma; n: number; HBV: hepatitis B virus; HCV: hepatitis C virus; TNM: tumor-node-metastasis; AFP: alpha-fetoprotein; CK19: cytokeratin 19.

Cox proportional hazards models were used in univariate and multivariate regression analyses. To control for stage, we stratified the patients into stage III/IV versus stage I/II, and compared survival within each group. We controlled for vascular invasion in the same way. The statistical computing language R 3.4.4 (The R Foundation, Vienna, Austria) was adopted for all the analyses. The statistical significance of the analyses was confirmed when a $\mathrm{P}$ value was less than 0.05 .

\section{Results}

\section{Immunostaining pattern of arginase- 1 and CK19}

In sections of HCC, arginase-1 positivity was demonstrated with cytoplasmic staining with or without nuclear staining within the tumor cells (Fig. 1a). Arginase-1 was highly ex- 

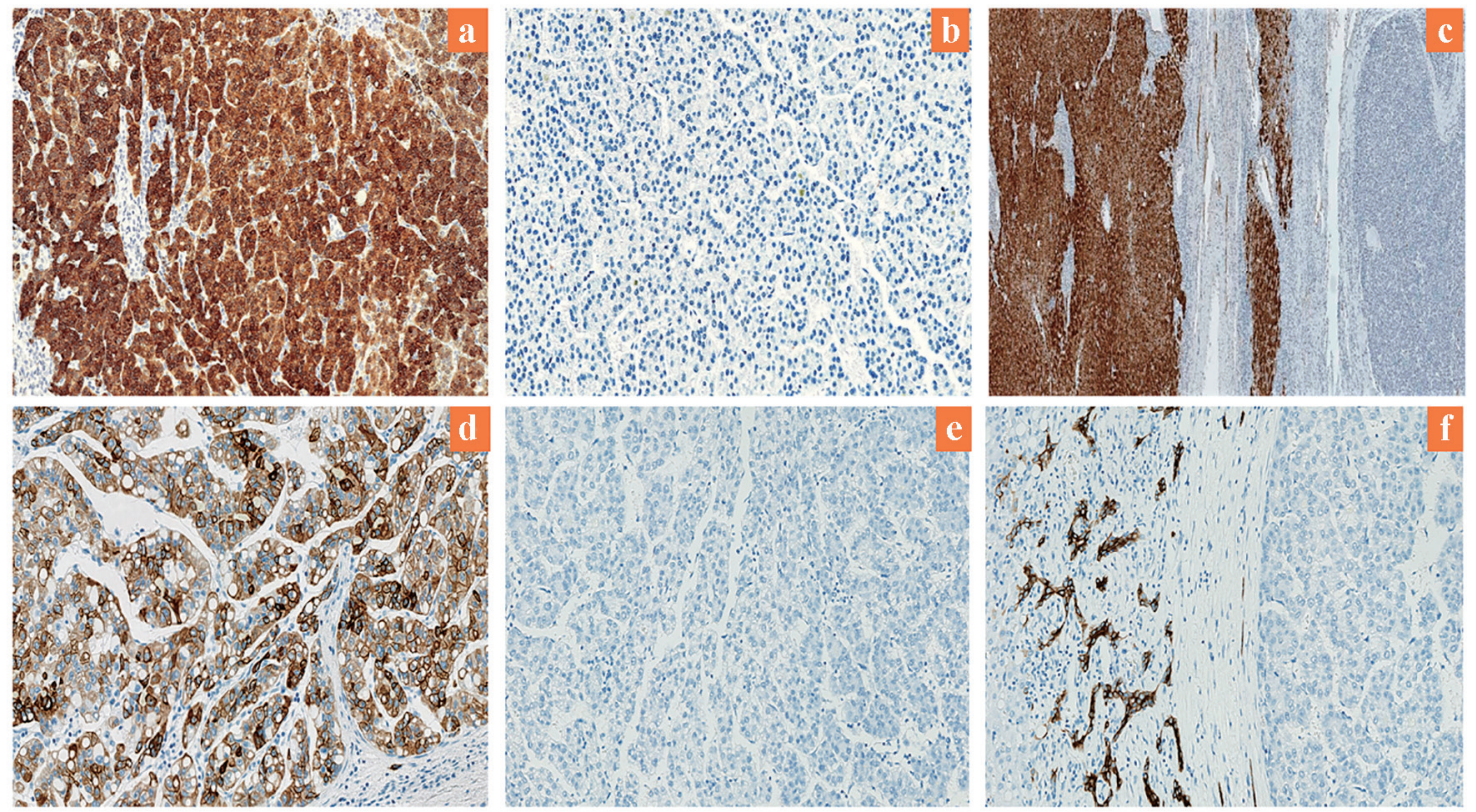

Figure 1. Expression of arginase-1 and CK19 in HCC. (a) High arginase-1 expression ( $\times 200)$. (b) Low arginase-1 expression (× 200). (c) Arginase-1 is highly expressed in adjacent benign liver $(\times 12.5)$. (d) Positive CK19 expression $(\times 200)$. (e) Negative CK19 expression $(\times 200)$. (f) CK19 expression in adjacent benign bile duct epithelium $(\times 200)$. CK19: cytokeratin 19; HCC: hepatocellular carcinoma.

pressed in 93 of the HCCs $(83 \%)$, showing immunostaining in $80-100 \%$ of those tumors. Of the 19 HCCs with low arginase- 1 expression, eight $(7.1 \%)$ showed negative staining for the protein (Fig. 1b), while the remaining patients had weak expression of arginase-1 (in $<15 \%$ of the tumor cells). Sections of adjacent benign liver demonstrated intense and diffuse staining in all the hepatocytes (Fig. 1c). CK19 expression was positive in the cytoplasm and membranes of hepatocytes in 19 HCCs (17\%) (Fig. 1d); all other HCCs were negative for CK19 (Fig. 1e). In addition, positive CK19 staining was observed in the positive control normal (adjacent benign bile duct epithelium) tissue (Fig. 1f).

\section{Clinicopathologic significance of arginase-1 and CK19}

Table 1 shows the statistical (positive) correlation of arginase-1 expression with hepatitis $\mathrm{B}$ viral infection $(\mathrm{P}=0.02)$, vascular invasion $(\mathrm{P}=0.044)$, and TNM stage $(\mathrm{P}=0.009)$. CK19 expression was not associated with any clinicopathologic variable. Other parameters, including tumor grade, tumor number, tumor size, presence of cirrhosis, serum AFP level, hepatitis $\mathrm{C}$ viral infection, age and sex showed no significant correlation with arginase-1 or CK19 expression.

\section{Prognostic significance of arginase-1 and CK19}

In the univariate analyses (Table 2), the clinicopathologic parameters of serum AFP level, tumor grade, vascular invasion and TNM stage were all statistically significant for adverse recurrence-free and overall survival (all, P < 0.05). CK19 expression in HCC was associated with decreased recurrencefree survival when compared with CK19-negative HCC (P $=0.001$ ), while arginase-1 expression was not (Fig. 2). The combination of arginase- 1 and CK19 expressions more significantly discriminated poor patient prognosis than either marker alone $(\mathrm{P}=0.0008)$.

Stratification of arginase-1 expression over the various TNM stages demonstrated that patients with high arginase-1 expression and advanced TNM stage were associated with lower recurrence-free survival $(\mathrm{P}=0.03)$, whereas the combination of CK19 expression and advanced TNM stage was not. Similarly, patients with high arginase-1 $(\mathrm{P}=0.04)$ or CK19 $(\mathrm{P}=0.007)$ expression and vascular invasion were negatively associated with recurrence-free survival. High arginase- 1 and CK19 expressions in addition to either TNM stage $(\mathrm{P}=0.01)$, multiple tumors $(\mathrm{P}=0.02)$ or vascular invasion $(\mathrm{P}=0.0008)$ significantly correlated with lower recurrence-free survival. However, neither arginase-1 nor CK19 was significantly associated with overall survival.

Multivariate analysis (Table 3) demonstrated that tumor grade was an independent poor prognostic indicator for both recurrence-free and overall survival $(\mathrm{P}=0.01$ and $\mathrm{P}=0.005$, respectively). CK19 expression was an independent prognostic predictor for only decreased recurrence-free survival $(\mathrm{P}$ $=0.003)$. When the combined evaluation of arginase- 1 and CK19 expressions was used as replacement of CK19 alone or arginase-1 alone, the combination variable was a significant independent predictor of postoperative recurrence $(\mathrm{P}=0.002)$.

Of the 112 total patients in our study, 30 (12 CK19-positive and 15 CK19-negative) developed recurrence during the 
Table 2. Univariate Predictors for RFS and OS in Patients With HCC

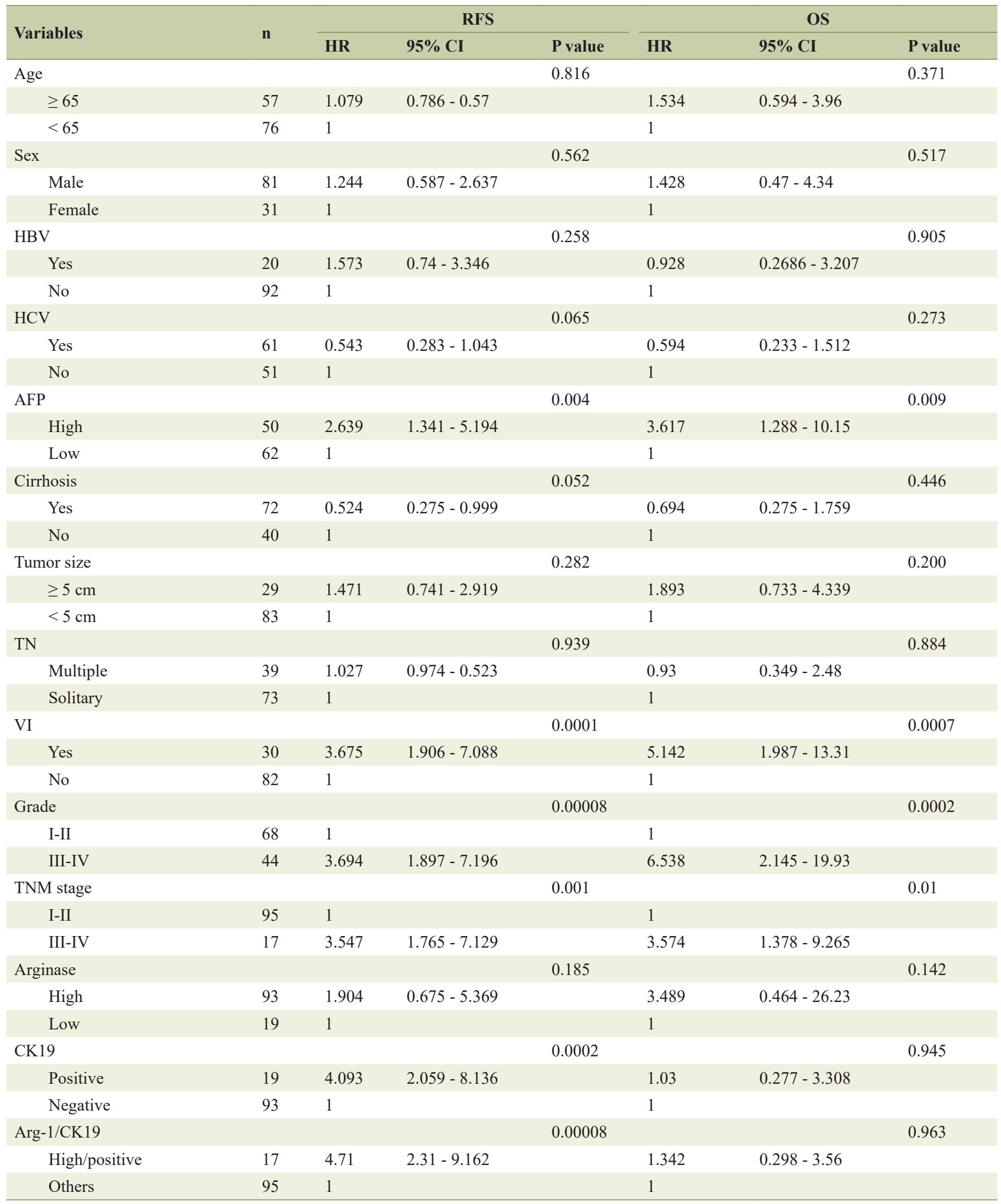


Table 2. Univariate Predictors for RFS and OS in Patients With HCC - (continued)

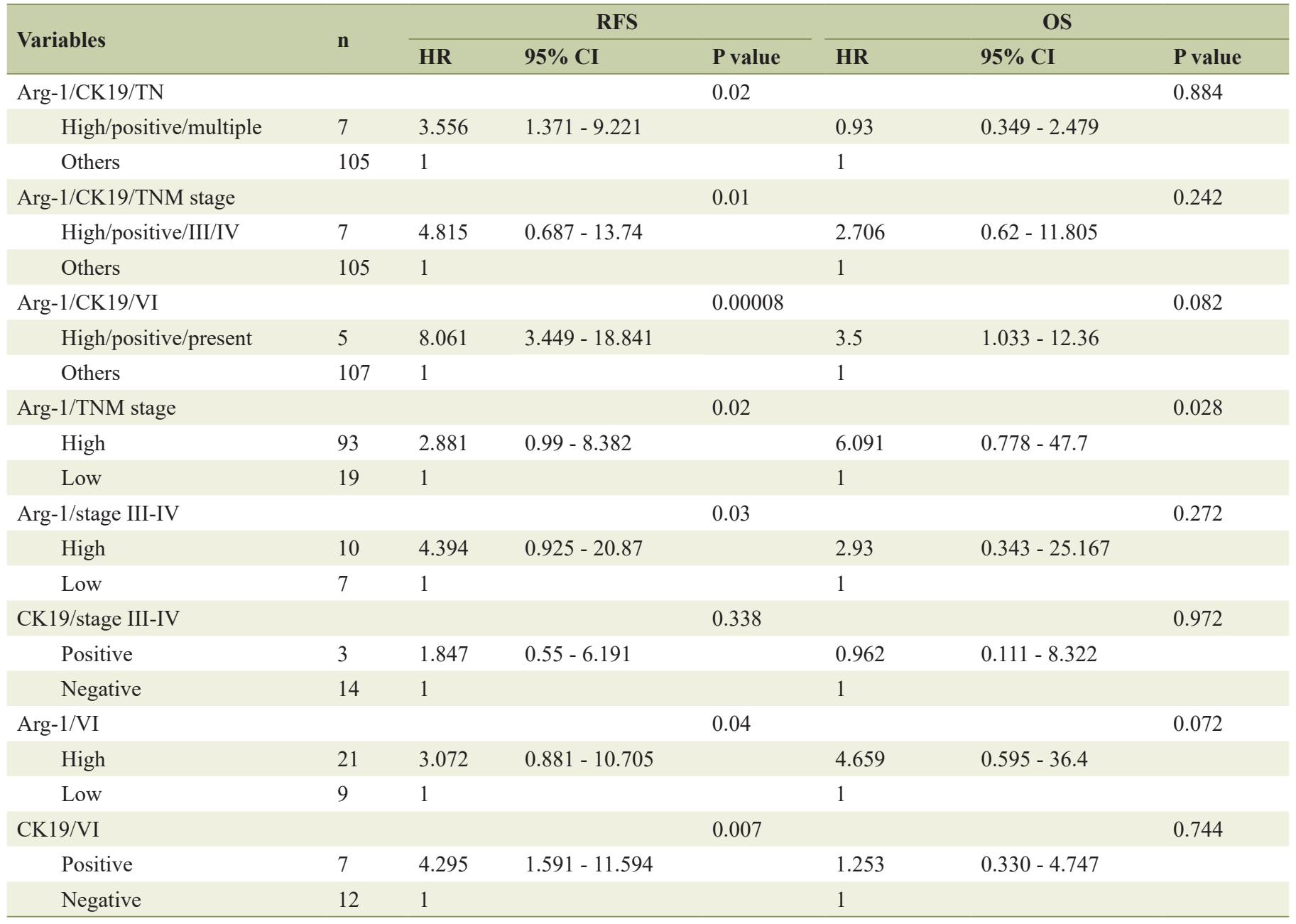

HCC: hepatocellular carcinoma; OS: overall survival; RFS: recurrence-free survival; HR: hazard ratio; Cl: confidence interval; HBV: hepatitis B virus; HCV: hepatitis C virus; VI: vascular invasion; TN: tumor number; TNM: tumor-node-metastasis; AFP: alpha-fetoprotein; Arg-1: arginase-1; CK19: cytokeratin 19.

follow-up period. The overall median time to recurrence was 12 months, while the overall median time to death was 12.5 months. Recurrence occurred in 23 patients with high arginase-1 HCC expression, and median time to recurrence was lower in patients with high arginase-1 HCC expression (12 months) than in those with low arginase-1 HCC expression (19 months). On the other hand, the median time to recurrence in patients with CK19-positive HCC was 10.5 months compared to 14.5 months in those with CK19-negative HCC.

\section{Discussion}

In the normal liver, hepatocytes express the cytokeratin family members CK8 and CK18 as well as a variety of hepatocytic markers, such as HepPar-1 and arginase-1; in contrast, CK19 is expressed in the cholangiocytes and hepatic progenitor cells [14]. HCC is typically derived from malignant transformation of benign hepatocytes and is characteristically associated with high expression of arginase-1. However, CK19 expression has been reported in HCC derived from hepatic stem cells $[11,14]$. Tumor grade, tumor size, tumor number, vascular invasion and TNM staging have all been used as predictors of recurrencefree survival. Favorable outcomes have been observed in patients who undergo curative surgery but recurrence of $\mathrm{HCC}$ can occur in patients with excellent tumor characteristics, such as well-differentiated tumors without vascular invasion. Thus, it is imperative that additional tumor markers for prediction of recurrence be identified.

CK19 expression has been associated with early recurrence $[12,16]$, but the prognostic significance requires further evaluation. Knowledge on the clinicopathologic and prognostic importance of arginase-1 in HCC is very limited currently. Furthermore, the combination of arginase-1 and CK19 as prognostic markers has never been explored. In this study, we evaluated and addressed these issues. The prognostic significance of arginase- 1 has only been analyzed in one study to date. Mao and colleagues [8] showed that high arginase-1 ex- 

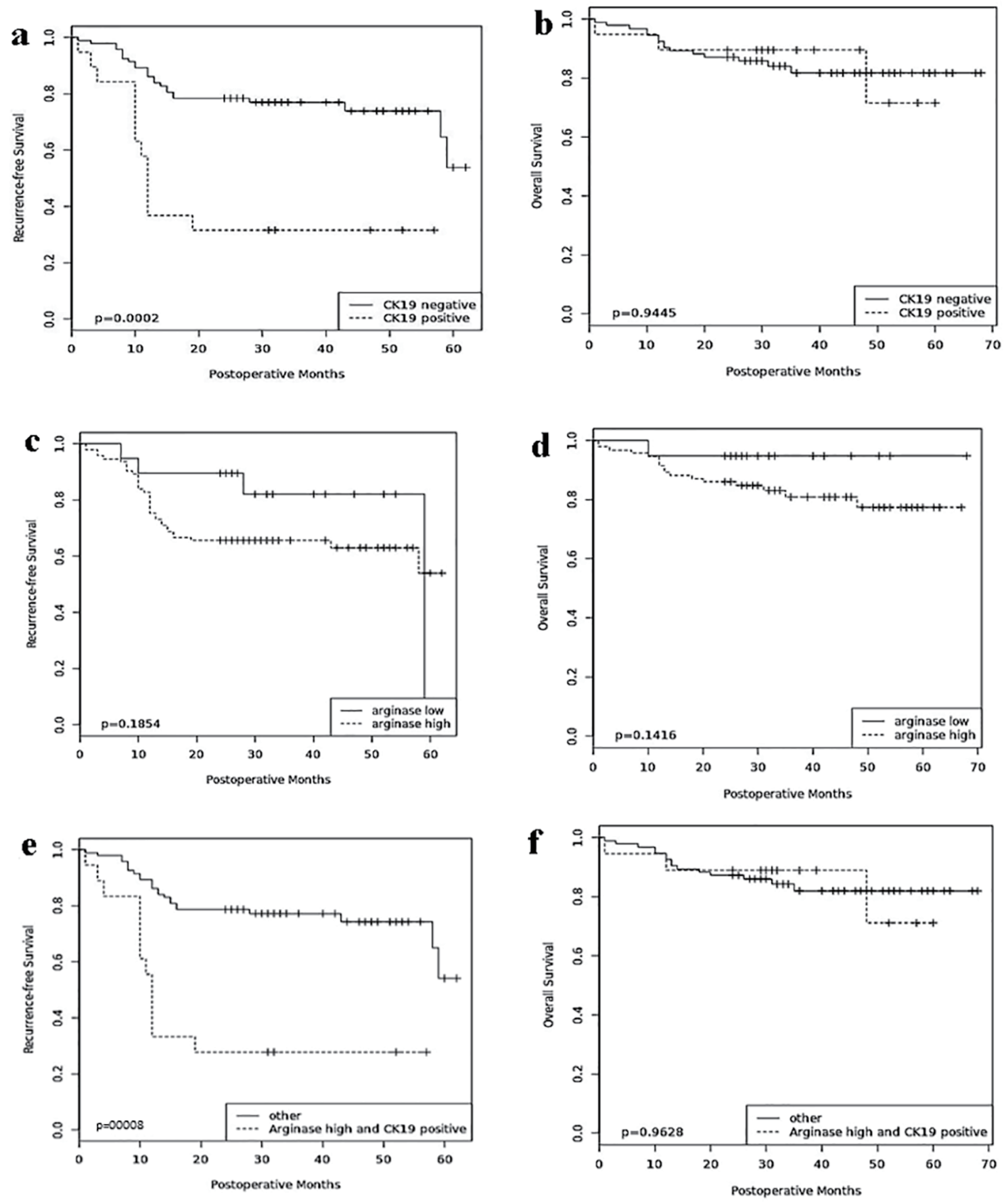

Figure 2. Prognostic significance of arginase-1 and CK19. Comparisons of recurrence-free survival and overall survival by CK19 (a, b), arginase-1 (c, d), and arginase-1/CK19 (e, f). CK19: cytokeratin 19.

pression was associated with higher recurrence-free and overall survival. However, $98 \%$ of the HCC tumors in that study showed arginase-1 expression, as detected by immunostaining with a polyclonal antibody, which may have increased nonspecific staining, making clear stratification of arginase-1 expression hard to interpret. In our study described herein, we used a highly specific monoclonal antibody that greatly im- proved detection of arginase-1. In our patients with low arginase expression, immunostaining for arginase-1 was either absent or had weak or focal staining in less than $15 \%$ of the tumor cells. Our patients with high arginase-1 expression had a shorter median time to recurrence than the patients with low arginase-1 expression. Interestingly, the patients with high arginase-1 expression also had higher hazard ratios (HRs) for 
Table 3. Multivariate Predictors for RFS and OS in Patients With HCC

\begin{tabular}{|c|c|c|c|c|c|c|c|c|}
\hline \multirow{2}{*}{ Variables } & \multicolumn{4}{|c|}{ RFS } & \multicolumn{4}{|c|}{ OS } \\
\hline & HR & $95 \%$ CI & & $P$ value & HR & $95 \% \mathrm{CI}$ & & $P$ value \\
\hline AFP & 1.629 & 0.613 & 0.7506 & 0.21 & 2.256 & 0.677 & 7.512 & 0.05856 \\
\hline Vascular invasion & 1.456 & 0.581 & 3.646 & 0.422 & 1.5537 & 0.4325 & 5.582 & 0.49947 \\
\hline TNM stage III/IV & 1.848 & 0.8172 & 4.178 & 0.14 & 1.495 & 0.4721 & 4.734 & 0.49414 \\
\hline
\end{tabular}

HCC: hepatocellular carcinoma; OS: overall survival; RFS, recurrence-free survival; HR: hazard ratio; Cl: confidence interval; TNM: tumor-nodemetastasis; AFP: alpha-fetoprotein; CK19: cytokeratin 19. ${ }^{*}$ This P value was obtained from replacement of single variable CK19 to the combined variable in the Cox regression analysis.

recurrence-free survival (HR: $1.904, \mathrm{P}=0.12$ ) and overall survival (HR: $3.489, \mathrm{P}=0.14$ ), although the values did not reach the level for statistical significance. In addition, we found the high arginase-1 expression to be associated with decreased recurrence-free survival in patients with vascular invasion and advanced TNM stage, suggesting its potential as a predictor for recurrence in these patients. The findings of this study are not surprising because the majority of HCC tumors express arginase-1, suggesting that it will be unusual to be identified as a good prognostic parameter in use alone. Limitations as to why statistical significance was not achieved include the small sample size. Secondly there was an uneven patient population into high and low arginase populations. This is attributed to the sensitivity and specificity of our monoclonal arginase- 1 antibody which is very efficient in the stratification of arginase-1 based on expression. Very few HCC tumors have low expression levels of arginase-1. These reasons might account, at least in part, for the imperfect results. Large-scale studies appear warranted for the evaluation of arginase-1 in HCC.

On the other hand, CK19 was found to be significantly associated with decreased recurrence-free survival (both in the univariate and multivariate analyses). We further showed that the combined evaluation of arginase-1 and CK19 was more effectively associated with lower recurrence-free survival in univariate analysis than arginase-1 alone (Table 2). Thus, CK19 might increase the prognostic power of arginase-1 in HCC patients, a notion which is further supported by the confirmatory univariate and multivariate analyses. Examination of the data suggests that this may be due to exclusion of arginase-1 low expression in the tumors that were CK19-positive. Furthermore, combinations of arginase- 1 and CK19 with other significant clinicopathologic factors were all associated with decreased recurrence-free survival. Although CK19 and CK19/ arginase-1 positive tumors were associated with decreased recurrence-free survival, they both did not achieve significant poor overall survival. We believe that this is due to small sample size which indicates that the analysis of overall survival has lower power when compared to that of recurrence-free survival. This is reflected in the HR which is slightly greater than 1 in both clinical factors, and perhaps in a larger study with a longer follow-up period, this upward trend will become significant. Secondly, the wide $95 \%$ confidence interval sup- ports this theory. Furthermore, the smaller effect of CK19 and $\mathrm{CK} /$ arginase- 1 on overall survival may be due to the fact that a median overall survival 2 - 4 times longer than recurrence-free survival is required for one to see any meaningful statistical significance. Lastly, the overall survival of the patients may be influenced by competing risk factors of death and possible treatment effect. Determining the true prognostic value of CK19 and CK19/arginase-1 in association with overall survival will require further evaluation in large clinical studies.

The study of arginase- 1 as a prognostic factor is very important. Arginase is responsible for the conversion of arginine to urea and L-ornithine in the urea cycle. Arginine, a semiessential amino acid, has been shown to be important for the proliferation of myriad human cancers, including HCC [17]. Arginine depletion inhibits the growth of human HCC through cell cycle arrest and apoptosis. Such arginine depletion in HCC can be achieved either by the use of pegylated arginine deaminase [18] or human recombinant arginase [19]. HCC is thought to be dependent on exogenous arginine for growth because the cells generally cannot internally synthesize arginine and are, therefore, auxotrophic for arginine. The mechanism of tumor inhibition through arginine depletion in HCC is thought to be related to the lack of argininosuccinate synthetase, the ratelimiting enzyme in arginine synthesis. Although arginine deprivation shows promising results in in vitro and in vivo studies, clear clinical benefits are yet to be demonstrated in human clinical trials in patients with advanced HCC [20-22]. Interestingly, since arginase is highly expressed in $\mathrm{HCC}$, it would be difficult to believe that it is associated with good prognosis. Indeed, arginase expression was found by western blotting to be significantly higher in HCC compared to normal and cirrhotic liver tissues [23]. Chrzanowska and coworkers [24, 25] showed high serum arginase activity in patients with HCC, which drastically decreased following surgery; therefore, the authors suggested a role for arginase in monitoring patients with HCC following hepatectomy which correlates well with our hypothesis.

These findings collectively suggest that arginase activity may be involved in the tumorigenesis of HCC. Because the mechanism of the metabolism of arginase in HCC is not clearly understood and the demonstration that arginase expression is not associated with good prognosis, it is important to 
consider that there may be other associated variables that may not favor arginase as a feasible target for arginine depletion (or function deprivation). It may be possible that arginase expression in combination with other clinicopathologic parameters such as CK19 expression, vascular invasion and advanced tumor stage facilitates tumor progression which results in tumor recurrence. Thus, the exact prognostic role of arginase-1 should be subjected to further validation in large-scale studies.

In conclusion, our data indicate that combination of CK19 with arginase-1 might have higher prognostic efficiency than arginase-1 or CK19 alone in patients with HCC who have undergone curative hepatectomy in the determination of tumor recurrence. However, this approach requires further validation and may prove to be beneficial in prognostic stratification of patients with HCCs, helping to achieve better clinical outcome.

\section{Acknowledgments}

None.

\section{Author Contributions}

JC and JG performed the immunohistochemical studies; IEO, $\mathrm{KK}$ and BP equally participated in the data collection and analysis; IEO and BK designed and coordinated the research study and wrote the manuscript.

\section{Funding}

Supported by no dedicated source of funding.

\section{Conflict of Interest}

All the authors have no conflict of interest to declare.

\section{Informed Consent}

Patients were not required to give informed consent to the study because the analysis used anonymized clinical data that were obtained through a retrospective review of charts.

\section{References}

1. Nanashima A, Omagari K, Tobinaga S, Shibata K, Sumida Y, Mine M, Morino S, et al. Comparative study of survival of patients with hepatocellular carcinoma predicted by different staging systems using multivariate analysis. Eur J Surg Oncol. 2005;31(8):882-890.

2. Tandon P, Garcia-Tsao G. Prognostic indicators in hepatocellular carcinoma: a systematic review of 72 studies. Liver Int. 2009;29(4):502-510.

3. Tateishi R, Yoshida H, Shiina S, Imamura H, Hasegawa
K, Teratani T, Obi S, et al. Proposal of a new prognostic model for hepatocellular carcinoma: an analysis of 403 patients. Gut. 2005;54(3):419-425.

4. Sekine S, Ogawa R, McManus MT, Kanai Y, Hebrok M. Dicer is required for proper liver zonation. J Pathol. 2009;219(3):365-372.

5. Multhaupt H, Fritz P, Schumacher K. Immunohistochemical localisation of arginase in human liver using monoclonal antibodies against human liver arginase. Histochemistry. 1987;87(5):465-470.

6. Radwan NA, Ahmed NS. The diagnostic value of arginase-1 immunostaining in differentiating hepatocellular carcinoma from metastatic carcinoma and cholangiocarcinoma as compared to HepPar-1. Diagn Pathol. 2012;7:149.

7. Yan BC, Gong C, Song J, Krausz T, Tretiakova M, Hyjek E, Al-Ahmadie H, et al. Arginase-1: a new immunohistochemical marker of hepatocytes and hepatocellular neoplasms. Am J Surg Pathol. 2010;34(8):1147-1154.

8. Mao H, Gao W, Lu G, Fang F, Teng L. Clinicopathological and prognostic implications of arginase expression in hepatocellular carcinoma. Clin Lab. 2013;59(1-2):37-43.

9. Schmelzer E, Wauthier E, Reid LM. The phenotypes of pluripotent human hepatic progenitors. Stem Cells. 2006;24(8):1852-1858.

10. Shiojiri N, Lemire JM, Fausto N. Cell lineages and oval cell progenitors in rat liver development. Cancer Res. 1991;51(10):2611-2620.

11. Turner R, Lozoya O, Wang Y, Cardinale V, Gaudio E, Alpini G, Mendel G, et al. Human hepatic stem cell and maturational liver lineage biology. Hepatology. 2011;53(3):1035-1045.

12. Uenishi T, Kubo S, Yamamoto T, Shuto T, Ogawa M, Tanaka H, Tanaka S, et al. Cytokeratin 19 expression in hepatocellular carcinoma predicts early postoperative recurrence. Cancer Sci. 2003;94(10):851-857.

13. Yang XR, Xu Y, Shi GM, Fan J, Zhou J, Ji Y, Sun HC, et al. Cytokeratin 10 and cytokeratin 19: predictive markers for poor prognosis in hepatocellular carcinoma patients after curative resection. Clin Cancer Res. 2008;14(12):38503859.

14. Durnez A, Verslype C, Nevens F, Fevery J, Aerts R, Pirenne J, Lesaffre E, et al. The clinicopathological and prognostic relevance of cytokeratin 7 and 19 expression in hepatocellular carcinoma. A possible progenitor cell origin. Histopathology. 2006;49(2):138-151.

15. Chan AC, Fan ST, Poon RT, Cheung TT, Chok KS, Chan SC, Lo CM. Evaluation of the seventh edition of the American Joint Committee on Cancer tumour-nodemetastasis (TNM) staging system for patients undergoing curative resection of hepatocellular carcinoma: implications for the development of a refined staging system. HPB (Oxford). 2013;15(6):439-448.

16. Wu PC, Fang JW, Lau VK, Lai CL, Lo CK, Lau JY. Classification of hepatocellular carcinoma according to hepatocellular and biliary differentiation markers. Clinical and biological implications. Am J Pathol. 1996;149(4):11671175.

17. Haines RJ, Pendleton LC, Eichler DC. Argininosuccinate 
synthase: at the center of arginine metabolism. Int J Biochem Mol Biol. 2011;2(1):8-23.

18. Ensor CM, Holtsberg FW, Bomalaski JS, Clark MA. Pegylated arginine deiminase (ADI-SS PEG20,000 mw) inhibits human melanomas and hepatocellular carcinomas in vitro and in vivo. Cancer Res. 2002;62(19):5443-5450.

19. Cheng PN, Lam TL, Lam WM, Tsui SM, Cheng AW, Lo WH, Leung YC. Pegylated recombinant human arginase (rhArg-peg5,000mw) inhibits the in vitro and in vivo proliferation of human hepatocellular carcinoma through arginine depletion. Cancer Res. 2007;67(1):309-317.

20. Yang TS, Lu SN, Chao Y, Sheen IS, Lin CC, Wang TE, Chen SC, et al. A randomised phase II study of pegylated arginine deiminase (ADI-PEG 20) in Asian advanced hepatocellular carcinoma patients. Br J Cancer. 2010;103(7):954-960.

21. Izzo F, Marra P, Beneduce G, Castello G, Vallone P, De Rosa V, Cremona F, et al. Pegylated arginine deiminase treatment of patients with unresectable hepatocellular carcinoma: results from phase I/II studies. J Clin Oncol. 2004;22(10):1815-1822.

22. Chow AK, Ng L, Sing Li H, Cheng CW, Lam CS, Yau TC, Cheng PN, et al. Anti-tumor efficacy of a recombinant human arginase in human hepatocellular carcinoma. Curr Cancer Drug Targets. 2012;12(9):1233-1243.

23. Chrzanowska A, Krawczyk M, Baranczyk-Kuzma A. Changes in arginase isoenzymes pattern in human hepatocellular carcinoma. Biochem Biophys Res Commun. 2008;377(2):337-340.

24. Chrzanowska A, Grabon W, Mielczarek-Puta M, Baranczyk-Kuzma A. Significance of arginase determination in body fluids of patients with hepatocellular carcinoma and liver cirrhosis before and after surgical treatment. Clin Biochem. 2014;47(12):1056-1059.

25. Chrzanowska A, Mielczarek-Puta M, Skwarek A, Krawczyk M, Baranczyk-Kuzma A. [Serum arginase activity in patients with liver cirrhosis and hepatocellular carcinoma]. Wiad Lek. 2007;60(5-6):215-218. 